\title{
Measurement of H2AX Phosphorylation as a Marker of lonizing Radiation Induced Cell Damage
}

\author{
Aida Muslimovic, Pegah Johansson and Ola Hammarsten* \\ Department of Clinical Chemistry and Transfusion Medicine, \\ The Institute of Biomedicine, The Sahlgrenska Academy at University of Gothenburg, \\ Sweden
}

\section{Introduction}

Cancer therapy often involves drugs or radiation, which induce DNA double-strand breaks (DSBs) and cause death of tumor cells. A dilemma with radiation and chemotherapy is the narrow therapeutic window and the fact that the sensitivity to DNA damage varies greatly in the human population, resulting in severe side effects and therapy-related deaths. Radiation and chemotherapy are usually planned during 1-3 weeks and delivered over a period of weeks to months. DNA damage testing of the patient could therefore be performed as part of the therapy planning. The level of DNA damage and DNA repair capacity could be measured after the first dose of chemotherapy or radiation, to allow personalized dosing during the following weeks of treatment. Induction of DSBs is followed by rapid formation of gamma-H2AX foci, with thousands of H2AX molecules being phosphorylated in the chromatin flanking the DSB site. Gamma-H2AX foci, each representing one radiation-induced DSB, can be measured by flow cytometry or counted in cell nuclei by fluorescence microscopy. In this chapter we will focus on radiation-induced DNA damage and its biological effects. We will also describe available methods for gamma$\mathrm{H} 2 \mathrm{AX}$ detection and discuss its possible use in clinical practice.

\section{Radiation-induced DNA damage}

The two DNA strands in the double-stranded DNA helix are over $20 \AA$ apart. Therefore, DNA-damaging chemicals like hydroxyl radicals or methylating agents for the most part generate single-stranded DNA damage, leaving the opposite strand intact. To take care of this type of DNA damage, human cells have several template-directed DNA repair systems, like nucleotide excision repair and base excision repair that require at least 10-30 nucleotides of an undamaged template strand to direct the synthesis of new DNA after removal of the DNA damage. These DNA repair systems are highly effective and accurate. Therefore, most forms of single-stranded DNA damage are removed within minutes without mutations. Single-stranded DNA damage is therefore not very toxic. As an example, over 100000

${ }^{*}$ Corresponding Author 
hydrogen peroxide-induced single-stranded DNA damage is required to affect the viability of a human cell (Muslimovic, Nystrom et al. 2009). However, template-directed repair is not possible when both DNA strands within 10-20 bp are damaged, called clustered DNA damage (Fig. 1).

\section{Non DSB-clusters}

\section{DSB-clusters}
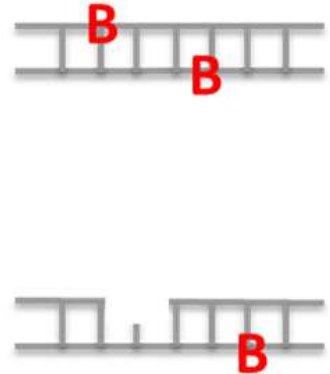

\section{Simple}

\section{Complex}
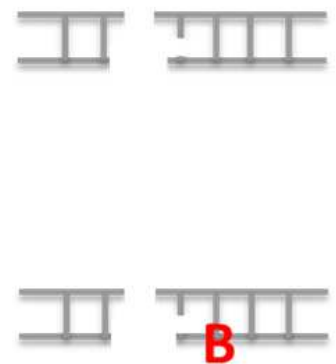

Fig. 1. Different types of radiation induced clustered DNA damage.

In this instance the cell has to repair the broken DNA by direct end joining (NHEJ) or homology-directed DNA repair (HR) (see next section) that are error prone and slow. Ionizing radiation is an efficient inducer of clustered DNA damage and is therefore highly toxic and mutagenic.

Ionizing photons or particles (ionizing radiation (IR)) damage DNA by direct energy deposition, leading to ionization events within the DNA molecule as well as ionization of water molecules and the formation of highly reactive hydroxyl radicals that damage the DNA indirectly. The passing photon or particle induces multiple ionizations as it passes through biological tissue and the number of such ionization events per unit of distance depends on the quality of the IR. High linear energy transfer (LET) IR induces denser ionization energy tracks than low LET IR. It is this ability to produce multiple ionization events within a short distance that is the reason behind the unique capacity of IR to generate damage to both DNA strands in close proximity (Hall 2000).

The clustered DNA damage induced by IR may include base damage to both strands, onestrand break opposed by base damage or the simple DNA double-strand break (DSB) with two strand beaks on opposing strands within 10-20 bp apart. In addition, the DSB can contain additional base damage close to the broken strands called complex clustered DSBs (Fig. 1). The complexity of the lesions increases with increasing LET of the radiation. For low LET, $30-40 \%$ of the DNA damage consists of complex clustered DSBs, as opposed to $90 \%$ for high LET (Nikjoo, O'Neill et al. 1999). Therefore, high LET IR is more toxic than low LET IR at the same absorbed dose.

IR induces ionization and molecular damage in all cellular components but it is its ability to damage DNA that is biologically important. For instance, it is possible to irradiate only parts of a cell using microscopic needle tips coated with alpha-emitting polonium, due to the short range of this radiation. In this setting, the mean lethal dose for a cell is $1.5 \mathrm{~Gy}$ if the irradiated area includes the nucleus, whereas the viability of the cell remains unaffected 
even after 250 Gy if only the cytoplasm is irradiated (Hall 2000). This clearly demonstrates that the biological effects of irradiation are due to its interaction with the nucleus. Furthermore, studies using selective irradiation of DNA by incorporation of radioactive nucleotides in cellular DNA have shown that DNA is the relevant biological target of IR.

Although 95\% of the DNA damage induced by IR is single-stranded DNA lesions, it is the few DSBs that are responsible for the toxic effects. Microinjection of more than 300 molecules of circular DNA with different forms of single-stranded DNA damage into the nucleus of human cells failed to induce significant cell cycle arrest, compared with injection of undamaged DNA. In contrast, nuclear injection of only 1-10 molecules of DNA containing one DSB per molecule were able to induce permanent cell cycle arrest (Huang, Clarkin et al. 1996), demonstrating that DSBs are particularly capable of inducing clonogenic cell death, the type of cell death typically induced by IR. Therefore, if we can measure the levels of DSBs induced after a dose of irradiation we will have a good measure of the biological effects of this dose.

\section{DSB repair}

In mammalian cells, there are two main pathways of DSB repair, homologous recombination (HR) and non-homologous end joining (NHEJ) (Mladenov and Iliakis; Mahaney, Meek et al. 2009). HR is the pathway of choice during the S and G2 phases when an identical sister chromatid, produced during the $S$ phase, can be used as a template for accurate DSB repair (Rothkamm, Kruger et al. 2003). Therefore, HR is considered to be an error-free repair pathway. However, since over $99 \%$ of the cells in a human body are in the G1 or G0 stage of the cell cycle, where HR is supressed, NHEJ will repair most of the DSBs induced by IR in humans. Most of the DSBs produced by IR can not be directly ligated, as they contain base damage and damage to the sugar phosphate backbone that must be processed prior to repair. Therefore the broken DNA ends are first trimmed and then ligated. As a consequence of the processing, nucleotides may be lost, generating deletion mutations at the breakpoint. Therefore, NHEJ is an error-prone DNA repair pathway, explaining the highly mutagenic nature of IR.

In canonical NHEJ, DSBs are repaired by a process involving DNA-dependent protein kinase, DNA-PK. Once a DSB is induced, $\mathrm{Ku}$, the DNA-binding subunit of DNA-PK, binds specifically to the DNA ends, due to a preformed channel in its structure that allows $\mathrm{Ku}$ to slide onto the end like a nut on a screw (Mimori and Hardin 1986; de Vries, van Driel et al. 1989; Walker, Corpina et al. 2001). Once bound to DNA ends, Ku will translocate inwards onto the DNA, allowing two DNA-PKcs molecules to bind to DNA ends (Hammarsten, DeFazio et al. 2000; DeFazio, Stansel et al. 2002) and interact in a synaptic complex across the DSB (DeFazio, Stansel et al. 2002), leading to activation of the DNA-PK holoenzyme (Meek, Gupta et al. 2004; Meek 2009) and subsequent autophosphorylation (Mahaney, Meek et al. 2009). DNA-PKcs binds to and activates the nuclease Artemis which is believed to be involved in the end processing of more complex DSBs (Ma, Pannicke et al. 2002; Wang, Pluth et al. 2005; Drouet, Frit et al. 2006; Goodarzi, Yu et al. 2006). Autophosphorylation is also thought to be required for the conformational change between opened and closed holoenzyme conformations, which allows end processing by polynucleotide kinase (PNK), polymerization by the DNA polymerases micro or gamma and finally ligation by LigIV/XRCC4/XLF complex. This DNA-PK dependent NHEJ pathway, dependent on DNA-PK is referred to as D-NHEJ, where D is short for DNA-PK or C-NHEJ, short for canonical NHEJ. 
Lately, a back-up NHEJ pathway (B-NHEJ) has been discussed in the literature. Studies where cells defective in HR and D-NHEJ were treated with DSB-inducing agents have revealed an alternative DSB-repair pathway with yet slower kinetics and with an increased number of chromosomal abberations, see review (Mladenov and Iliakis). In this pathway, PARP1 binds to DNA ends instead of to $\mathrm{Ku}$ and the LigIII/XRCC1 complex performs the ligation.

DSBs induced by IR are repaired by different kinetics. After high doses of IR, repair of $85 \%$ of all DSBs occur during the first 10-30 minutes, whereas the remaining $15 \%$ of the breaks can take up to 24 hours to be fully removed (Goodarzi, Jeggo et al.). This dual kinetic profile probably reflects a different chemical complexity at the DSB ends and the local chromatin compaction. In agreement with this possibility, complex DSBs induced by high LET and DSBs induced in heterochromatin are repaired more slowly, whereas a larger portion of low LET-induced DSBs and DSBs in low-complexity euchromatic regions are repaired with fast kinetics (Goodarzi, Jeggo et al.). In G1 cells, all components of the NHEJ are required for both slow and fast DSB-repair. An exception is Artemis, which probably is only required for the slow repair of more complex lesions. The slow repair of DSBs in high-complexity heterochromatin regions also involve ATM/p-KAP1, the MRN complex, gamma-H2AX, MDC1, RNF8, RNF168 and 53BP1 (Goodarzi, Jeggo et al.). It is likely that DSBs with different chemical modifications require distinct repair complexes involving polymerase, polynucleotide kinase and nuclease activities. As we still lack a reliable in vitro system for DSB repair and methods to induce DSBs with a defined chemical structure in cells, our knowledge of how different repair complexes assemble in response to different forms of DSBs is still incomplete.

\section{DSB response and $\mathrm{H} 2 \mathrm{AX}$ phosphorylation}

The DNA of eukaryotic cells is organized in DNA protein complexes called chromatin. Chromatin proteins mostly consist of histones, small arginine-lysine-rich proteins with a high binding affinity for the negatively charged DNA. Chromatin is organized in smaller structural subunits called nucleosomes. The bead particles called nucleosome core particles contain about 150bp DNA coiled 1.7 times around the core consisting of eight histones, two from each of the $\mathrm{H} 2 \mathrm{~A}, \mathrm{H} 2 \mathrm{~B}, \mathrm{H} 3$ and $\mathrm{H} 4$ types, and, additional 50bp DNA for the linkerhistone $\mathrm{H} 1$ connecting two nucleosomes. The nucleosome structure results in a packaging of DNA into $10 \mathrm{~nm}$ fibers, which can be further compacted into $30 \mathrm{~nm}$ fibers and chromatin loops.

One of the first cellular responses to DSBs is the rapid phosphorylation of histone $\mathrm{H} 2 \mathrm{AX}$ (Rogakou, Pilch et al. 1998). H2AX is one of the most evolutionary conserved H2A variants and constitutes $2-25 \%$ of all nucleosomes, depending on the cell type (Kinner, Wu et al. 2008). H2AX phosphorylation occurs on serine- 139 in the SQ motif that is positioned at the carboxy terminus of H2AX (Rogakou, Pilch et al. 1998; Rogakou, Boon et al. 1999). H2AX phosphorylation occurs minutes after DSB induction, leading to the formation of distinct gamma-H2AX foci (Rogakou, Boon et al. 1999). Several reports indicate that each IR-induced gamma-H2AX focus represents a single DSB, at least in G1 cells (Sedelnikova, Rogakou et al. 2002; Pilch, Sedelnikova et al. 2003; Rothkamm and Lobrich 2003). The level of gamma$\mathrm{H} 2 \mathrm{AX}$ induced by different drugs and radiation is closely correlated to the level of cell death (Banath and Olive 2003; Muslimovic, Nystrom et al. 2009). This indicates that measurement of gamma-H2AX in patient cells will be a biologically important marker for the induction of DSBs and toxicity following chemotherapy and radiotherapy. 
Ataxia-telangiectasia-mutated (ATM), DNA-dependent protein kinase (DNA-PK) and Rad3-related (ATR) kinases that belong to the phosphatidylinositol-OH-kinase-like family of protein kinases (PIKKs), can phosphorylate H2AX (Paull, Rogakou et al. 2000; Ward and Chen 2001; Burma, Chen et al. 2002; Furuta, Takemura et al. 2003; Stiff, O'Driscoll et al. 2004; Ward, Minn et al. 2004). ATM and DNA-PK phosphorylate H2AX redundantly upon DSB induction in the G1 and G2 phases of the cell cycle (Stiff, O'Driscoll et al. 2004), while ATR is responsible for replication stress and UV damage related $\mathrm{H} 2 \mathrm{AX}$ phosphorylation during the S phase (Ward and Chen 2001; Ward, Minn et al. 2004).

The first event in the recognition of a newly formed DSB is the binding of a complex of three proteins, Mre11, Rad50 and Nbs1 (MRN) to the DNA ends. The MRN complex is a multifunctional DNA-binding protein complex with a weak preference for DNA ends, a nuclease function (Mre11) and the ability to recruit ATM dimers to the DNA via the C terminus of Nbs1. In this setting, ATM dimers are activated for their kinase activity. Active ATM monomers then phosphorylate H2AX close to the end, which leads to recruitment of mediator of DNA damage checkpoint protein 1 (MDC1), a protein that binds specifically to gamma-H2AX. As MDC1 also binds to the MRN complex, this leads to a positive feed-back loop where additional MRN complexes and ATM are recruited to the DSB site, resulting in extended H2AX phosphorylation by ATM (see below).

Initially, gamma-H2AX foci are formed as small, distinct foci at the site of the DSB, visible as soon as 1-3 minutes after irradiation. With time, these gamma-H2AX foci grow and spread along the chromatin, further away from the DSB site, and reach a plateau after 10-30 minutes (Rogakou, Pilch et al. 1998; Rogakou, Boon et al. 1999; Sedelnikova, Rogakou et al. 2002). Depending on the celline, $0.03-0.06 \%$ of H2AX molecules per DSB become phosphorylated and spread over $2 \mathrm{Mbp}$ regions of chromatin that covers $\sim 2000$ gammaH2AX molecules (Rogakou, Pilch et al. 1998). However, the phosphorylated gamma-H2AX regions can reach up to $30 \mathrm{Mbp}$ from the break (Rogakou, Boon et al. 1999; Pilch, Sedelnikova et al. 2003). In this way, the DSB signal is locally amplified by the spreading of the $\mathrm{H} 2 \mathrm{AX}$ phosphorylation to large chromatin regions. Several reports have described large residual gamma-H2AX foci that form several hours after irradiation (Bocker and Iliakis 2006; Markova, Schultz et al. 2007; Yamauchi, Oka et al. 2008; Yoshikawa, Kashino et al. 2009) and these foci can persist for months (Rodier, Coppe et al. 2009).

When DSBs are repaired, H2AX phosphorylation declines with time. Dephosphorylation of $\mathrm{H} 2 \mathrm{AX}$ has been proposed as the possible mechanism. Four serine/threonine phosphatases have been reported to dephosphorylate H2AX in human cells: PP2A, PP4, PP6 and PP2C. PP2A dephosphorylates H2AX at the site of the DSB and siRNA knockdown of the catalytic subunit of PP2A leads to persistence of foci and inefficient DSB repair (Chowdhury, Keogh et al. 2005). PP4 associates with chromatin and dephosphorylates chromatin-bound H2AX molecules phosphorylated by ATR during replication (Douglas, Zhong et al. ; Chowdhury, Xu et al. 2008; Nakada, Chen et al. 2008). PP6 also dephosphorylates H2AX (Douglas, Zhong et al.) and knockdown of its catalytic subunit results in persistent gamma-H2AX and 53BP1 foci. PP2A and PP6 seem to be involved in the recovery from the G2/M checkpoint. PPC2 has been co-purified with $\mathrm{H} 2 \mathrm{~A}-\mathrm{H} 2 \mathrm{~B}$ histone dimer and may be involved in the dephosphorylation of dissociated $\mathrm{H} 2 \mathrm{~A}-\mathrm{H} 2 \mathrm{~B}$ and the incorporation of the unphosphorylated H2A-H2B into the nucleosome (Kimura, Takizawa et al. 2006). Recently, WIP1 (wild-type53-induced phosphatase) WIP1 has been reported to dephosphorylate H2AX, resulting in suppression of the DSB repair (Macurek, Lindqvist et al.; Moon, Nguyen et al.). 


\section{Gamma-H2AX detection and its usage}

Measurements of gamma-H2AX phosphorylation are currently the most sensitive way to detect IR-induced DNA damage and to measure the extent of DSB repair. Measurements of gamma-H2AX foci could therefore be a possible approach to monitor DSBs or DSB repair in cancer patients during treatment with radiotherapy or chemotherapy as a way to personalize the dosing. It is also possible that gamma-H2AX measurements could be used for radiation biodosimetry for nuclear plant workers or in the general public after nuclear accidents as well as exposure to potentially DNA damaging environmental toxins. In the following sections we will summarize the methods that have been developed for gammaH2AX measurement in humans and its possible future use in cancer treatment and public dosimetric testing.

\section{Methods for measurement of gamma-H2AX in patient cells}

\subsection{General considerations}

One major consideration in gamma-H2AX foci biodosimetry is the source of patient cells to be assayed after exposure to DNA-damaging agents.

Peripheral blood mononuclear cells prepared by density gradient centrifugation are perhaps the optimal source of cells for this purpose, for several reasons. Firstly, over a million cells can routinely be obtained from each milliliter of blood and prepared within 30 minutes by a simple centrifugation step. Secondly, the acquisition is not as invasive as obtaining biopsies and poses little risk to patients. Finally, the possibility of quick sampling post-radiation is important for the gamma-H2AX assay, due to the fast repair kinetics of foci. A potential problem with blood mononuclear cells is that at least three different cell types are present in these preparations. The most prominent cell type is lymphocytes $(80-95 \%$ T-lymphocytes and $5-20 \%$ B-lymphocytes) that constitute $80-90 \%$ of the cells. In addition, the mononuclear cell preparation contains monocytes $(5-10 \%)$ and a variable amount of contaminating neutrophiles. All these cells are able to induce gamma-H2AX in response to IR, although the level of the gamma-H2AX response is lower in neutrophiles (Ismail, Wadhra et al. 2007) and in some subsets of lymphocytes (Andrievski and Wilkins 2009).

Another viable option that has been tested is hair bulb cells from plucked hair (Redon, Nakamura et al.). The sheath around the base of a plucked hair contains proliferating cells that are easily accessible. The hairs are collected by gripping them close to the skin using blunt-nosed forceps and plucking them with a single, sharp action, checking them for the presence of sheath and immersing them directly in a fixation medium. In humans, scalp hair bulbs have been shown to contain more viable cells than hair bulbs from the eyebrow. In a study using macaque monkeys receiving total body irradiation, hair bulbs from the fur did not contain enough cells for analysis, indicating that body hair from humans might not be useful. This study also showed that mononuclear cells yielded more reproducible results for the gamma-H2AX analysis than cells from hair bulbs. A problem with hair bulb cells is that this cell type can only be used for gamma-H2AX measurements following systemic treatment, unless the scalp or eyebrows have been part of the irradiated area. On the other hand, active hair bulbs contain proliferating cells as opposed to mononuclear cell preparations. This opens up the possibility of using cells from hair bulbs to monitor DNA damage induced by nucleoside analogues and other drugs that induce DNA damage only in proliferating cells. 


\subsection{Gamma-H2AX detection in patients with immunofluorescence microscopy}

The most sensitive method for gamma-H2AX detection is immunofluorescence microscopy, which enables quantification of single foci in individual cells. In this method, fixed cells or tissue are attached to glass slides and incubated with gamma-H2AX antibodies, usually overnight. After washing, the slides are stained with secondary antibodies and DNA dyes. After a final wash, the slides are dried and mounted with cover glasses before analysis by fluorescence microscopy (Fig. 2).
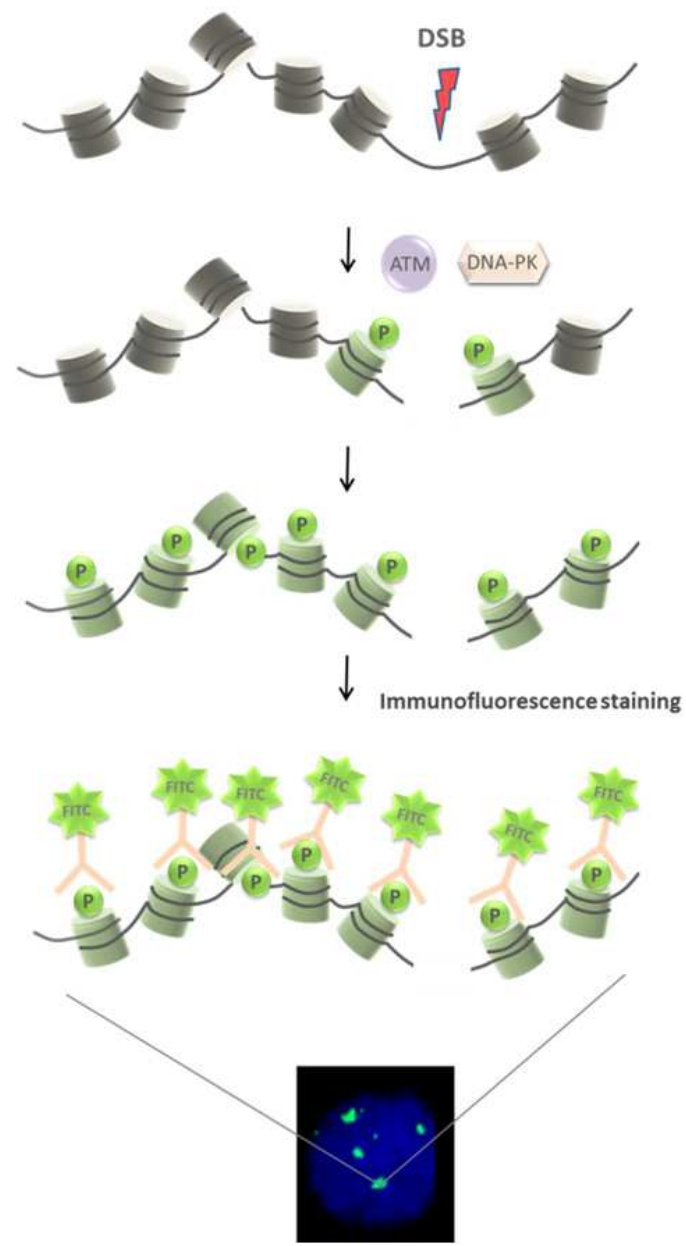

Fig. 2. Immunofluorescence analysis of $\mathrm{H} 2 \mathrm{AX}$ phosphorylation.

Gamma-H2AX are then quantified either by manual counting in fluorescence microscope or by foci counting software from digital images. Both methods of counting are errorprone, especially at high IR doses. A general problem is that gamma-H2AX foci are heterogeneous with large and small foci coexisting side by side in the same nucleus (Fig. 3 , white arrows). 


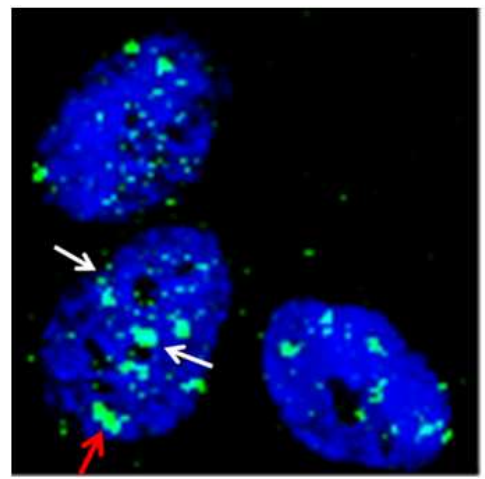

Fig. 3. Heterogeneous sizes of gamma-H2AX foci.

In addition, foci that are close together can be incorrectly scored as a single large focus (Fig. 3 , red arrow). This problem is especially prominent following high-LET irradiation, where multiple closely spaced DSBs are generated along the high-LET particle track. The problem of superimposed foci increases as the IR dose increases and limits foci counting to doses below 2-3 Gy where 20-40 foci are found in many nuclei. In addition, the number of foci per cell is often highly variable due to the distributive nature of DSB induction also when cells have been irradiated in vitro. After local irradiation of patients blood cells from the irradiated area are mixed with unirradiated cells, further adding to the heterogeneity. Therefore, over 50 cells must be counted to allow reliable measurements of gamma-H2AX induction in an in vitro-irradiated sample. In samples of mononuclear cells from patients receiving local irradiation, even more cells must be scored for reliable results. The requirement to count foci in a large number of nuclei in each sample makes the immunofluorescence microscopy method labor-intensive when the counting is done manually. On the other hand, spot-finding software have difficulties with the quantification settings, so that foci of different sizes and brightness are sometimes counted incorrectly. In addition, digital images are usually taken in a single plane where only a subset of the nuclei is in perfect focus, affecting the focus brightness and size. Lastly, background staining from debris in the sample may affect the counting when digital systems are used. However, microscopic systems dedicated to foci counting are now able to overcome many of these problems (Lobrich, Rief et al. 2005). These microscopic systems are currently expensive and rarely available in routine clinical laboratories.

Another problem with immunofluorescence microscopy is the handling of microscopic slides. Lymphocytes are normally attached to the microscopic slides via centrifugation (cytospin) that only allows one patient sample per slide. In addition, the staining of microscopic slides is time-consuming and requires large volumes of antibody solutions, adding to the analysis time and cost. We have therefore developed a modification of the gamma-H2AX immunofluorescence method, where fixed cells are stained with the fluorescent gamma-H2AX antibody in solution before being attached to microscopic slides by drying a droplet of the cell sample in a salt-free protein solution. This allows arraying of up to 16 patient samples per slide ready for foci counting in two hours (Johansson, Muslimovic et al. 2011). Together with automatic microscopic foci counting systems that are available (Lobrich, Rief et al. 2005), this new staining method holds great promise in future implementation of the gamma-H2AX foci analysis in clinical routine. 
Immunofluorescence microscopy and foci counting has been used both in vitro and in patients to measure DNA-damage following radiotherapy and chemotherapy. Initial studies using low doses of irradiation of human fibroblast cultures showed that there was a correlation between the IR dose, the numbers of gamma-H2AX foci, and amount of DSBs. This study also established that increased levels of gamma-H2AX foci are detectable at doses as low as 1mGy (Rothkamm and Lobrich 2003). The ability to detect very low levels of radiation has inspired the use of gamma-H2AX foci in mononuclear cells from patients exposed to X-rays in computerized tomography (CT) (Lobrich, Rief et al. 2005) and other X-ray examinations. Gamma-H2AX foci analysis was used as a biomarker for individual radiation damage in pediatric patients undergoing cardiac catherization showing that previous estimates according to linear-no-treshold hypothesis have been underestimating radiation damage. Therefore, gamma-H2AX foci analysis could be used as a tool for estimations of DNA-damage effects after cardiac catherization (Beels, Bacher et al. 2009). Another study showed that the amount of gamma-H2AX foci in lymphocytes after coronary CT angiography correlates to dose-length product (DLP) which is a physical parameter used to calculate delivered dose after CT. Therefore, gamma-H2AX foci analysis could be used to measure individual dose-related effects of X-rays (Kuefner, Grudzenski et al.). Gamma-H2AX foci analysis has also been used for estimations of individual radiation doses by comparisons of in vivo and in vitro exposed lymphocytes to fractionated irradiation or angiography indicating that gamma-H2AX foci analysis could be used for dose estimations (Kuefner, Grudzenski et al. 2009). In addition, several reports now show that gamma-H2AX foci analysis can be used to measure DNAdamage induced by local radiotherapy. Analysis of gamma-H2AX foci in prostate cancer biopsies after in vivo radiation showed reproducible quantifications of foci-numbers in the prostata specimens in the dose region 0-1Gy (Qvarnstrom, Simonsson et al. 2004). GammaH2AX foci has also been be used to follow induction of DNA-damage in lymphocytes of cancer patients with different cancer types and exposed to local radiotherapy treatments to different sites of the body. There was a correlation between mean number of gamma-H2AX foci per lymphocyte and integrated total body radiation dose indicating that measurements of gamma-H2AX foci in lymphocytes can be used for in vivo dosimetry (Sak, Grehl et al. 2007). Immunofluorescence microscopy has also been successful in the detection of a DSB response after treatment with cancer drugs, like the Topoisomerase-I inhibitor camptothecin (Kinders, Hollingshead et al.), in human biopsies (Qvarnstrom, Simonsson et al. 2004), circulating tumor cells (Wang, Pfister et al.), lymphocytes and plucked hair bulbs (Redon, Nakamura et al.). A fully automated high-throughput system, the RABIT (Rapid Automated Biodosimetry Tool), has been developed for gamma-H2AX analysis on human lymphocytes able to process several thousands of blood samples per day that might be useful in larger nuclear accidents (Garty, Chen et al.). Finally, it was reported that radioimmunoconjugates with a peptide that allows cellular uptake of labeled gamma-H2AX antibody complexes could be used to measure gamma-H2AX foci in living cells and in whole mice. The method was less sensitive than conventional gamma-H2AX foci staining of fixed and permeabilized cells, but this technique opens up the interesting possibility of monitoring DSB induction in patient tumors by imaging techniques, such as gamma camera or even MRT, in the future (Cornelissen, Kersemans et al.). While quantification of the gamma-H2AX foci is clearly sensitive enough to allow reliable measurement of DSB induction in patients, the method is still too labor-intensive, timeconsuming and operator-dependent. In addition, automatic fluorescence microscopes equipped with software for automatic counting of foci are rarely available in routine clinical laboratories. Therefore, the gamma-H2AX foci method is currently difficult to implement in clinical routine. 


\subsection{Gamma-H2AX detection in patients using flow cytometry}

Flow cytometry has also been used to measure gamma-H2AX phosphorylation in patient cells. In this method, the possibility to detect single gamma-H2AX foci and DSBs is lost. Instead, the gamma-H2AX phosphorylation signal from the whole cell is measured in a large number of cells. The flow cytometry method is less labor-intensive but, also less sensitive compared to counting gamma-H2AX foci by immunofluorescence microscopy.

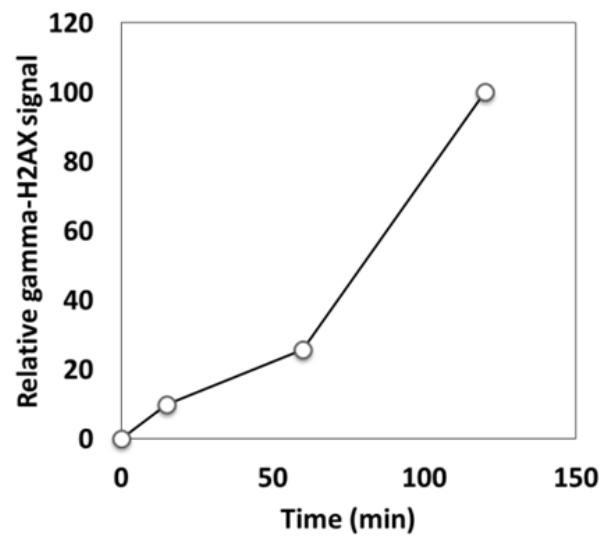

Fig. 4. Staining-time dependent gamma-H2AX signal analysed by flow cytometry.

In the flow cytometry method, cells are stained and permeabilized in a staining buffer containing fluorescently labeled gamma-H2AX antibodies and DNA dyes. After staining for 15 minutes up to a few hours (Fig. 4), the samples can be analyzed directly by flow cytometry without further processing. In flow cytometry, up to 100000 cells can be analyzed in each sample within minutes, allowing detection of even small subsets of gamma-H2AXpositive cells.

Due to its simplicity, this method can be implemented in its current form in clinical laboratories where flow cytometers often are available. An important shortcoming of this method is its low analytical sensitivity compared to counting of separate gamma-H2AX foci (Fig. 5).

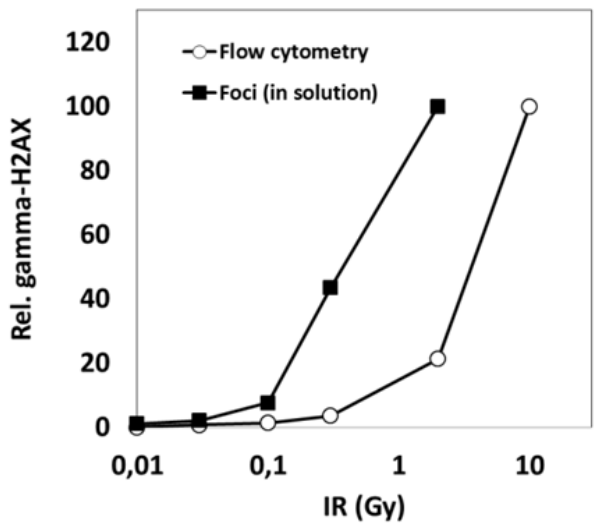

Fig. 5. Sensitivity of the gamma-H2AX detection by foci method and flow cytometry method. 
A contributing factor to the low sensitivity is that undamaged cells have a diffuse background signal from the gamma-H2AX antibodies. The nature of this background is unclear but results in a requirement of at least 2-5 gamma-H2AX foci per cell to separate the signal from undamaged cells. At low IR doses $(<0.1 \mathrm{~Gy})$ most cells contain $0-1$ foci per nucleus. The sensitivity of the foci method relies on its ability to score cells with one focus among cells without foci. Radiation doses generating an increase from one focus in every 20 cells ( $0.05 \mathrm{foci} / \mathrm{cell})$ to one focus in every 10 cells $(0.1 \mathrm{foci} / \mathrm{cell})$ is a significant increase that can be scored with the foci method. In contrast, this increase remains below the detection limit in the flow cytometer, as the gamma-H2AX signal from cells with 1-2 foci is not separated from undamaged cells. For that reason, flow cytometry analysis is only able to measure gamma-H2AX signal at IR doses of over 0.1-0.3 Gy, whereas the foci method is able to measure in the mGy range (Rothkamm and Lobrich 2003) and in our hands down to 0.01Gy (Fig. 6).

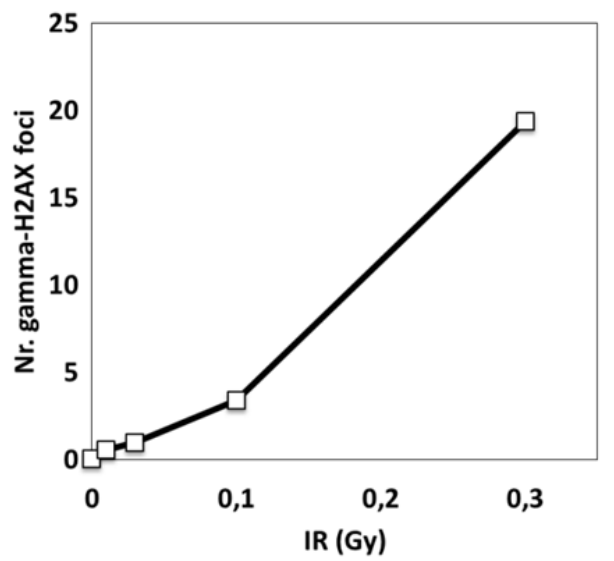

Fig. 6. Dose-dependence of gamma-H2AX foci formation in human lymphocytes after in vitro irradiation as measured by immunofluorescence microscopy with "in-solution" staining.

On the other hand, most radiotherapy regimens include fractionated doses of 1.5-5 Gy. For the subset of mononuclear cells residing in the irradiated area, this dose is well within the measurable range for flow cytometry analysis. In addition, flow cytometry allows analysis of small subsets of gamma-H2AX-positive cells, as up to 100000 cells are analyzed in each sample. This is particularly helpful when mononuclear cells are analyzed from patients following local irradiation, where blood cells from the irradiated area are mixed with unirradiated cells. In one study, blood samples were collected from three rectal cancer patients before and at different times after 5Gy pelvic irradiation (Muslimovic, Ismail et al. 2008). The gamma-H2AX signal was analyzed by flow cytometry and compared to in vitroirradiated mononuclear cells from the same patients. This analysis revealed that approximately $5 \%$ of the mononuclear cells retrieved one hour after the pelvic irradiation had the same gamma-H2AX signal as cells irradiated with 5 Gy in vitro. Apparently, these "sentinel cells" were trapped in the microcirculation in the irradiated area and therefore 
received the full 5 Gy dose. This pilot study indicated that flow cytometry may be particularly well suited for the measurement of gamma-H2AX induction in blood cells following local radiotherapy. The same flow cytometry method was tested on in vitroirradiated blood samples and found to be robust, reproducible and able to detect radiation doses as low as 0.1Gy with a linear increase of the gamma-H2AX signal at doses over $0.3 \mathrm{~Gy}$. The signal was specific for H2AX and was absent in mouse embryonic cells from H2AX-/mice. All nucleated blood cells displayed the gamma-H2AX signal following DSB induction. However, the signal was close to $50 \%$ lower from neutrophiles compared to monocytes and lymphocytes. In addition, the study revealed a marked inter-individual difference of the gamma-H2AX signal intensity following DSB-induction in vitro, with a twofold difference in gamma-H2AX signal intensity between the highest and lowest individual tested. This finding has been confirmed in other studies using similar methods (Bourton, Plowman et al.).

Flow cytometry measurement of gamma-H2AX has been used to diagnose Ataxia telangiectasia, a disease where patients are radiosensitive and show a delayed clearance of H2AX foci after in vitro irradiation of lymphocytes (Porcedda, Turinetto et al. 2008). Similar methods have been used to find patients with severe normal tissue toxicity following radiotherapy (Bourton, Plowman et al.) and to study the gamma-H2AX response in several subsets of nucleated blood cells (Andrievski and Wilkins 2009). However, apart from the pilot study of rectal cancer patients receiving pelvic irradation, no clinical study has used flow cytometry to measure gamma-H2AX levels in patient samples after radiotherapy. Future studies will reveal the usefulness of flow cytometry methods for gamma-H2AX measurement in the clinical setting.

\subsection{Other methods for gamma-H2AX measurement}

Gamma-H2AX analysis has been used in genotoxicity measurements with ELISA-based assays (Matsuzaki, Harada et al.) and high content screening assays (Yip, Cuddy et al.).

\section{Internal controls and standards}

Like all clinical laboratory methods, measurements of gamma-H2AX by flow cytometry or by immunofluorescence microscopy are subject to day-to-day variations due to many factors including different quality among antibody batches and other reagents, fluctuations in detector performance and staining efficiency. In addition, inter-laboratory variations in foci detection software and flow cytometry detector sensitivity must also be calibrated to allow data comparison between clinical laboratories. Therefore, in order to implement H2AX methods in clinical practice there will be a need for calibrators and internal controls to monitor method performance. For that purpose, we have developed two types of controls based on phosphopeptide coated plastic beads and fixed cells. Control beads are generated by binding biotinylated phosphopetides derived from $\mathrm{H} 2 \mathrm{AX}$ to streptavidin coated plastic beads, dried and stored at room temperature. Control cells are generated from primary human fibroblasts treated with the DSB inducing drug calicheamicin- $\gamma 1$ for 30 minutes followed by fixation and storage in $90 \% \mathrm{EtOH}$ at $-70{ }^{\circ} \mathrm{C}$. Both controls are stable over 40 days and are equally capable of correcting flow cytometry based gamma-H2AX signal from two different antibody batches (Muslimovic A., manuscript in preparation). These two types 
of controls have their own merits. The phosphopeptide coated beads are easy to manufacture in large quantities and can be shipped at ambient temperature facilitating inter-laboratory comparisons. A potential problem with the beads is that they do not present the antigen in a way that resembles the cellular structure in fixed cells. This could generate problems if phosphopeptide coated beads are used to harmonize phospho-H2AX methods using different antibodies with variable antigen specificity. We therefore also established conditions that allowed fixed cells to serve as controls. Control cells are less amenable to large scale production and must be shipped on dry-ice that could make inter-laboratory harmonization impractical. However, these control cells can also be used as controls for gamma-H2AX foci staining as the foci morphology and brightness are preserved during storage (Johansson 2011) (Fig. 7). Thus, control cells can be used in the foci method and added to each microscopic slide with patient samples as an internal control as well as interlaboratory comparisons of the gamma-H2AX foci method.

A

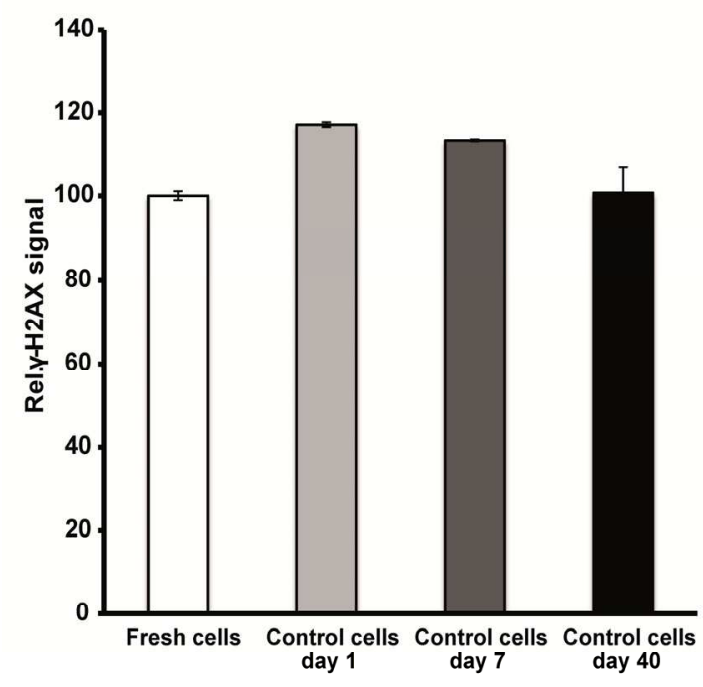

B

Fig. 7. A: Control cells in ethanol solution are stable up to 40 days and $-70^{\circ} \mathrm{C}$ as shown by flow cytometry. B: Control cells treated with different levels of DSB-inducing drug CLM preserve intact gamma-H2AX foci after storage in $-70^{\circ} \mathrm{C}$ and ethanol.

\section{Conclusion}

Gamma-H2AX analysis has enhanced our ability to detect DNA damage in individual patients and opens for the first time the possibility to measure DNA-damage during cancer 
treatment and in bio-monitoring purposes in clinical laboratories. In addition, a wide range of genotoxic events can now be monitored in individuals with the gamma-H2AX methods due to its unique sensitivity and tight correlation to the toxicity of the DNA-damage. In some cases there is substantial induction of gamma-H2AX foci with exposure to high levels of ionizing radiation and chemotherapeutic drugs that can be monitored with simple flow cytometry methods. In other instances, the DSB induction is of smaller magnitude, such as with diagnostic $\mathrm{CT}$ scans or exposure to environmental toxins, that can be measured using the more sensitive gamma-H2AX foci method. In addition, gamma-H2AX measurements can also be used following nuclear accidents, allowing rapid identification of individuals exposed to critically high radiation doses for initial triage and medical treatment decisions. There is, however, a need to further elucidate the molecular steps leading to gamma-H2AX formation to allow safe use of the technique. In addition, the technical limitations of the foci method need to be solved so that this method could be implemented in routine clinical labs. Alternatively, the sensitivity of the flow cytometry methods should be enhanced to allow detection of cells with single foci. When these limitations are solved the gamma-H2AX methods holds great promise as an important asset to allow reliable measurement of DNAdamage in clinical routine.

\section{References}

Andrievski, A. and R. C. Wilkins (2009). "The response of gamma-H2AX in human lymphocytes and lymphocytes subsets measured in whole blood cultures." Int J Radiat Biol 85(4): 369-76.

Banath, J. P. and P. L. Olive (2003). "Expression of phosphorylated histone H2AX as a surrogate of cell killing by drugs that create DNA double-strand breaks." Cancer Res 63(15): 4347-50.

Beels, L., K. Bacher, et al. (2009). "gamma-H2AX foci as a biomarker for patient X-ray exposure in pediatric cardiac catheterization: are we underestimating radiation risks?" Circulation 120(19): 1903-9.

Bocker, W. and G. Iliakis (2006). "Computational Methods for analysis of foci: validation for radiation-induced gamma-H2AX foci in human cells." Radiat Res 165(1): 113-24.

Bourton, E. C., P. N. Plowman, et al. "Prolonged expression of the gamma-H2AX DNA repair biomarker correlates with excess acute and chronic toxicity from radiotherapy treatment." Int J Cancer.

Burma, S., B. P. Chen, et al. (2002). "ATM Phosphorylates Histone H2AX in Response to DNA Double-strand Breaks*." J Biol Chem 276(Nov 9): 42462-7.

Chowdhury, D., M. C. Keogh, et al. (2005). "gamma-H2AX dephosphorylation by protein phosphatase 2A facilitates DNA double-strand break repair." Mol Cell 20(5): 801-9.

Chowdhury, D., X. Xu, et al. (2008). "A PP4-phosphatase complex dephosphorylates gamma-H2AX generated during DNA replication." Mol Cell 31(1): 33-46.

Cornelissen, B., V. Kersemans, et al. "Imaging DNA damage in vivo using gammaH2AXtargeted immunoconjugates." Cancer Res 71(13): 4539-49.

de Vries, E., W. van Driel, et al. (1989). "HeLa nuclear protein recognizing DNA termini and translocating on DNA forming a regular DNA-multimeric protein complex." J Mol Biol 208(1): 65-78. 
DeFazio, L. G., R. M. Stansel, et al. (2002). "Synapsis of DNA ends by DNA-dependent protein kinase." EMBO J 21(12): 3192-200.

Douglas, P., J. Zhong, et al. "Protein phosphatase 6 interacts with the DNA-dependent protein kinase catalytic subunit and dephosphorylates gamma-H2AX." Mol Cell Biol 30(6): 1368-81.

Drouet, J., P. Frit, et al. (2006). "Interplay between Ku, Artemis, and the DNA-dependent protein kinase catalytic subunit at DNA ends." J Biol Chem 281(38): 27784-93.

Furuta, T., H. Takemura, et al. (2003). "Phosphorylation of histone H2AX and activation of Mre11, Rad50, and Nbs1 in response to replication-dependent DNA double-strand breaks induced by mammalian DNA topoisomerase I cleavage complexes." J Biol Chem 278(22): 20303-12.

Garty, G., Y. Chen, et al. "The RABIT: a rapid automated biodosimetry tool for radiological triage." Health Phys 98(2): 209-17.

Goodarzi, A. A., P. Jeggo, et al. "The influence of heterochromatin on DNA double strand break repair: Getting the strong, silent type to relax." DNA Repair (Amst) 9(12): 1273-82.

Goodarzi, A. A., Y. Yu, et al. (2006). "DNA-PK autophosphorylation facilitates Artemis endonuclease activity." EMBO J 25(16): 3880-9.

Hall, Ë. J. (2000). Radiobiology for the Radiologist. Philadelphia, PA, Lippincott Williams \& Wilkins.

Hammarsten, O., L. G. DeFazio, et al. (2000). "Activation of DNA-dependent protein kinase by single-stranded DNA ends." J Biol Chem 275(3): 1541-50.

Huang, L. C., K. C. Clarkin, et al. (1996). "Sensitivity and selectivity of the DNA damage sensor responsible for activating p53-dependent G1 arrest." Proc Natl Acad Sci U S A 93(10): 4827-32.

Ismail, I. H., T. I. Wadhra, et al. (2007). "An optimized method for detecting gamma-H2AX in blood cells reveals a significant interindividual variation in the gamma-H2AX response among humans." Nucleic Acids Res 35(5): e36.

Johansson, P., A. Muslimovic, et al. "In-solution staining and arraying method for the immunofluorescence detection of gammaH2AX foci optimized for clinical applications." Biotechniques 51(3): 185-9.

Kimura, H., N. Takizawa, et al. (2006). "A novel histone exchange factor, protein phosphatase 2Cgamma, mediates the exchange and dephosphorylation of H2AH2B." J Cell Biol 175(3): 389-400.

Kinders, R. J., M. Hollingshead, et al. "Development of a validated immunofluorescence assay for gammaH2AX as a pharmacodynamic marker of topoisomerase I inhibitor activity." Clin Cancer Res 16(22): 5447-57.

Kinner, A., W. Wu, et al. (2008). "Gamma-H2AX in recognition and signaling of DNA double-strand breaks in the context of chromatin." Nucleic Acids Res 36(17): 5678-94.

Kuefner, M. A., S. Grudzenski, et al. "Effect of CT scan protocols on x-ray-induced DNA double-strand breaks in blood lymphocytes of patients undergoing coronary CT angiography." Eur Radiol 20(12): 2917-24. 
Kuefner, M. A., S. Grudzenski, et al. (2009). "DNA double-strand breaks and their repair in blood lymphocytes of patients undergoing angiographic procedures." Invest Radiol 44(8): 440-6.

Lobrich, M., N. Rief, et al. (2005). "In vivo formation and repair of DNA double-strand breaks after computed tomography examinations." Proc Natl Acad Sci U S A 102(25): 8984-9.

Ma, Y., U. Pannicke, et al. (2002). "Hairpin opening and overhang processing by an Artemis/DNA-dependent protein kinase complex in nonhomologous end joining and V(D)J recombination." Cell 108(6): 781-94.

Macurek, L., A. Lindqvist, et al. "Wip1 phosphatase is associated with chromatin and dephosphorylates gammaH2AX to promote checkpoint inhibition." Oncogene 29(15): 2281-91.

Mahaney, B. L., K. Meek, et al. (2009). "Repair of ionizing radiation-induced DNA doublestrand breaks by non-homologous end-joining." Biochem J 417(3): 639-50.

Markova, E., N. Schultz, et al. (2007). "Kinetics and dose-response of residual 53BP1/gamma-H2AX foci: co-localization, relationship with DSB repair and clonogenic survival." Int J Radiat Biol 83(5): 319-29.

Matsuzaki, K., A. Harada, et al. "Whole cell-ELISA to measure the gammaH2AX response of six aneugens and eight DNA-damaging chemicals." Mutat Res 700(1-2): 71-9.

Meek, K. (2009). "New targets to translate DNA-PK signals." Cell Cycle 8(23): 3809.

Meek, K., S. Gupta, et al. (2004). "The DNA-dependent protein kinase: the director at the end." Immunol Rev 200: 132-41.

Mimori, T. and J. A. Hardin (1986). "Mechanism of interaction between Ku protein and DNA." J Biol Chem 261(22): 10375-9.

Mladenov, E. and G. Iliakis "Induction and repair of DNA double strand breaks: the increasing spectrum of non-homologous end joining pathways." Mutat Res 711(1-2): 61-72.

Moon, S. H., T. A. Nguyen, et al. "Dephosphorylation of gamma-H2AX by WIP1: an important homeostatic regulatory event in DNA repair and cell cycle control." Cell Cycle 9(11): 2092-6.

Muslimovic, A., I. H. Ismail, et al. (2008). "An optimized method for measurement of gamma-H2AX in blood mononuclear and cultured cells." Nat Protoc 3(7): 1187-93.

Muslimovic, A., S. Nystrom, et al. (2009). "Numerical analysis of etoposide induced DNA breaks." PLoS One 4(6): e5859.

Nakada, S., G. I. Chen, et al. (2008). "PP4 is a gamma H2AX phosphatase required for recovery from the DNA damage checkpoint." EMBO Rep 9(10): 1019-26.

Nikjoo, H., P. O'Neill, et al. (1999). "Quantitative modelling of DNA damage using Monte Carlo track structure method." Radiat Environ Biophys 38(1): 31-8.

Paull, T. T., E. P. Rogakou, et al. (2000). "A critical role for histone H2AX in recruitment of repair factors to nuclear foci after DNA damage." Curr Biol 10(15): 886-95.

Pilch, D. R., O. A. Sedelnikova, et al. (2003). "Characteristics of gamma-H2AX foci at DNA double-strand breaks sites." Biochem Cell Biol 81(3): 123-9. 
Porcedda, P., V. Turinetto, et al. (2008). "A rapid flow cytometry test based on histone H2AX phosphorylation for the sensitive and specific diagnosis of ataxia telangiectasia." Cytometry A 73(6): 508-16.

Qvarnstrom, O. F., M. Simonsson, et al. (2004). "DNA double strand break quantification in skin biopsies." Radiother Oncol 72(3): 311-7.

Redon, C. E., A. J. Nakamura, et al. "The use of gamma-H2AX as a biodosimeter for totalbody radiation exposure in non-human primates." PLoS One 5(11): e15544.

Rodier, F., J. P. Coppe, et al. (2009). "Persistent DNA damage signalling triggers senescenceassociated inflammatory cytokine secretion." Nat Cell Biol 11(8): 973-9.

Rogakou, E. P., C. Boon, et al. (1999). "Megabase chromatin domains involved in DNA double-strand breaks in vivo." J Cell Biol 146(5): 905-16.

Rogakou, E. P., D. R. Pilch, et al. (1998). "DNA double-stranded breaks induce histone H2AX phosphorylation on serine 139." J Biol Chem 273(10): 5858-68.

Rothkamm, K., I. Kruger, et al. (2003). "Pathways of DNA double-strand break repair during the mammalian cell cycle." Mol Cell Biol 23(16): 5706-15.

Rothkamm, K. and M. Lobrich (2003). "Evidence for a lack of DNA double-strand break repair in human cells exposed to very low x-ray doses." Proc Natl Acad Sci U S A 100(9): 5057-62.

Sak, A., S. Grehl, et al. (2007). "gamma-H2AX foci formation in peripheral blood lymphocytes of tumor patients after local radiotherapy to different sites of the body: dependence on the dose-distribution, irradiated site and time from start of treatment." Int J Radiat Biol 83(10): 639-52.

Sedelnikova, O. A., E. P. Rogakou, et al. (2002). "Quantitative detection of (125)IdUinduced DNA double-strand breaks with gamma-H2AX antibody." Radiat Res 158(4): 486-92.

Stiff, T., M. O'Driscoll, et al. (2004). "ATM and DNA-PK function redundantly to phosphorylate H2AX after exposure to ionizing radiation." Cancer Res 64(7): 2390-6.

Walker, J. R., R. A. Corpina, et al. (2001). "Structure of the Ku heterodimer bound to DNA and its implications for double-strand break repair." Nature 412(6847): 607-14.

Wang, J., J. M. Pluth, et al. (2005). "Artemis deficiency confers a DNA double-strand break repair defect and Artemis phosphorylation status is altered by DNA damage and cell cycle progression." DNA Repair (Amst) 4(5): 556-70.

Wang, L. H., T. D. Pfister, et al. "Monitoring drug-induced gammaH2AX as a pharmacodynamic biomarker in individual circulating tumor cells." Clin Cancer Res 16(3): 1073-84.

Ward, I. M. and J. Chen (2001). "Histone H2AX is phosphorylated in an ATR-dependent manner in response to replicational stress." J Biol Chem 276(51): 47759-62.

Ward, I. M., K. Minn, et al. (2004). "UV-induced ataxia-telangiectasia-mutated and Rad3related (ATR) activation requires replication stress." J Biol Chem 279(11): 9677-80.

Yamauchi, M., Y. Oka, et al. (2008). "Growth of persistent foci of DNA damage checkpoint factors is essential for amplification of G1 checkpoint signaling." DNA Repair (Amst) 7(3): 405-17. 
Yip, K. W., M. Cuddy, et al. "A high-content screening (HCS) assay for the identification of chemical inducers of PML oncogenic domains (PODs)." J Biomol Screen 16(2): 251-8.

Yoshikawa, T., G. Kashino, et al. (2009). "Phosphorylated H2AX foci in tumor cells have no correlation with their radiation sensitivities." J Radiat Res (Tokyo) 50(2): 151-60. 


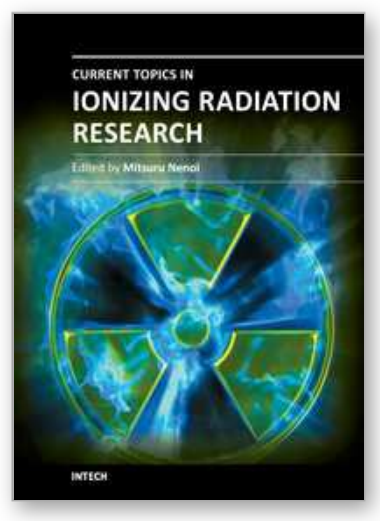

\author{
Current Topics in lonizing Radiation Research \\ Edited by Dr. Mitsuru Nenoi
}

ISBN 978-953-51-0196-3

Hard cover, 840 pages

Publisher InTech

Published online 12, February, 2012

Published in print edition February, 2012

Since the discovery of $X$ rays by Roentgen in 1895 , the ionizing radiation has been extensively utilized in a variety of medical and industrial applications. However people have shortly recognized its harmful aspects through inadvertent uses. Subsequently people experienced nuclear power plant accidents in Chernobyl and Fukushima, which taught us that the risk of ionizing radiation is closely and seriously involved in the modern society. In this circumstance, it becomes increasingly important that more scientists, engineers and students get familiar with ionizing radiation research regardless of the research field they are working. Based on this idea, the book "Current Topics in lonizing Radiation Research" was designed to overview the recent achievements in ionizing radiation research including biological effects, medical uses and principles of radiation measurement.

\title{
How to reference
}

In order to correctly reference this scholarly work, feel free to copy and paste the following:

Aida Muslimovic, Pegah Johansson and Ola Hammarsten (2012). Measurement of H2AX Phosphorylation as a Marker of lonizing Radiation Induced Cell Damage, Current Topics in Ionizing Radiation Research, Dr. Mitsuru Nenoi (Ed.), ISBN: 978-953-51-0196-3, InTech, Available from: http://www.intechopen.com/books/currenttopics-in-ionizing-radiation-research/measurement-of-h2ax-phosphorylation-as-a-marker-of-ionizing-radiationinduced-cell-damage

\section{INTECH}

open science | open minds

\section{InTech Europe}

University Campus STeP Ri

Slavka Krautzeka 83/A

51000 Rijeka, Croatia

Phone: +385 (51) 770447

Fax: +385 (51) 686166

www.intechopen.com

\section{InTech China}

Unit 405, Office Block, Hotel Equatorial Shanghai

No.65, Yan An Road (West), Shanghai, 200040, China

中国上海市延安西路65号上海国际贵都大饭店办公楼 405 单元

Phone: +86-21-62489820

Fax: $+86-21-62489821$ 
(C) 2012 The Author(s). Licensee IntechOpen. This is an open access article distributed under the terms of the Creative Commons Attribution 3.0 License, which permits unrestricted use, distribution, and reproduction in any medium, provided the original work is properly cited. 